\title{
DOTAP:Cholesterol-Fus1 Liposome Complex
}

National Cancer Institute

\section{Source}

National Cancer Institute. DOTAP:Cholesterol-Fus1 Liposome Complex. NCI Thesaurus. Code C91708.

A formulation composed of DOT AP:cholesterol liposomal nanoparticles complexed with a plasmid expression cassette encoding human FUS1 protein, with potential antineoplastic activity. Upon administration, DOT AP:chol-Fus1 liposome complex accumulates mainly in the lungs and particularly in cancer cells. Upon transfer of the Fus 1 gene into tumor cells, the expression of Fus1 may induce tumor cell apoptosis and suppress tumor cell proliferation. Fus1, a potent tumor-suppressor protein, is present in normal, healthy cells but often absent in certain cancer cells. DOTAP:cholesterol liposome is composed of cationic lipid dioleoyl-trimethylammonium propane (DOTAP) and cholesterol at a molar ratio of 1:1. 